\title{
Histopathologic Analysis of Atypical Lesions in Image- Guided Core Breast Biopsies
}

\author{
Michelle Bonnett, M.D., Tracie Wallis, B.S., Michelle Rossmann, M.D., Nat L. Pernick, M.D., \\ David Bouwman, M.D., Kathryn A. Carolin, M.D., Daniel Visscher, M.D. \\ Departments of Pathology (MB, TW), Surgery (DB, KAC), and Radiology (MR), Karmanos Cancer Institute \\ and Wayne State University School of Medicine, Detroit, Michigan; Department of Pathology (DV), Mayo \\ Clinic, Rochester, Minnesota; and PathologyOutlines.com, LLC (NLP), Bingham Farms, Michigan
}

Appropriate follow-up of patients with needle core breast biopsies (NCBB) showing atypical hyperplasia remains unclear because previous studies show that subsequent open biopsies in variable proportions of these patients reveal ductal carcinoma in situ (DCIS) or even invasive carcinoma, indicating significant sampling artifact. NCBB with diagnoses of atypia were morphologically classified into groups as follows: I, ALH $(n=24)$; II, ADH with minimal cytologic atypism ( $n=90)$; III, atypia, other (9 columnar, 2 apocrine, 11 atypical papillary); IV, severe ADH/borderline DCIS $(n=31)$. Mammographic and histologic features, including the number of foci of atypia in the NCBB and the calcification span, were then correlated with presence of DCIS or invasive tumor in subsequent open excisions. Open excisional biopsies showed more severe lesions in $12 \%$ of Group I-III cases $(8 \%$ in Group I, 9\% in Group II, and 27\% in Group III), of which 15 were DCIS and one was an invasive tubular carcinoma $(0.3 \mathrm{~cm})$. Of the DCIS, $60 \%(n=9)$ were $\leq 5 \mathrm{~mm}$, and 13 of $15(87 \%)$ were low grade. The NCBB cavity was immediately adjacent to the more severe lesions in $88 \%(n=14)$ of cases, in keeping with sampling error. The subset showing severe ADH with borderline nuclear features in contrast was associated with a high likelihood $(63 \%)$ of DCIS in follow-up excisions. NCBB with atypical papillary features also showed a high frequency of DCIS $(4 / 11,36 \%)$ in subsequent open excisions. Other factors associated with more severe lesions on open biopsy included the number of atypical foci in the NCBB $(>4, P<.05)$ and the mammographic calcification span $(>2.0 \mathrm{~cm}, P<.0001)$. Atypical

\footnotetext{
Copyright (C) 2003 by The United States and Canadian Academy of Pathology, Inc.

VOL. 16, NO. 2, P. 154, 2003 Printed in the U.S.A

Date of acceptance: December 3, 2002.

Address reprint requests to: Daniel Visscher, M.D., Department of Pathology, Mayo Clinic, 200 First St. SW, Rochester, MN 55905; fax: 507-2841599; e-mail: visscher.daniel@mayo.edu.

DOI: 10.1097/01.MP.0000052375.72841.E2
}

lesions diagnosed in NCBB samples are radiographically and morphologically heterogeneous, accounting for the variable frequency of DCIS or invasive neoplasm identified in subsequent open excisions, which are usually focal, low grade, and a consequence of sampling artifact (i.e., adjacent to the NCBB cavity). DCIS is more likely if microcalcifications are mammographically extensive or if atypia is multifocal or is associated with borderline cytologic features.

KEY WORDS: Atypical hyperplasia, Ductal carcinoma in situ, Stereotactic breast biopsy.

Mod Pathol 2003;16(2):154-160

Stereotactic or ultrasound-guided needle core breast biopsies (NCBB) are now routinely performed to assess suspicious microcalcifications or non-palpable masses detected by screening mammography. This approach has been shown to have high sensitivity and specificity in establishing the diagnosis of fibrocystic lesions, ductal carcinoma in situ (DCIS), and invasive carcinoma (1). As more NCBB are being performed, pathologists are encountering lesions with features of atypical ductal hyperplasia $(\mathrm{ADH})$ or atypical lobular hyperplasia (ALH). There are two issues that impact the clinical evaluation of patients with atypical lesions in NCBB samples. First, malignant lesions are known to frequently coexist with ADH/ALH, either within the same segment of breast tissue or in different quadrants (the latter as a manifestation of so-called field effect alteration). Because of partial/limited sampling, it is thus theoretically possible that at least some patients with atypia on NCBB may harbor occult DCIS. This problem is highlighted by the consideration that histologic distinction between $\mathrm{ADH}$ and DCIS is partially quantitative in nature (2, $3)$. Second, microfocal lesions may be missed during the process of trimming the block (so-called sectioning artifact). This problem is exacerbated if cores are fragmented or associated with crushing, 
which may preclude full morphologic evaluation of lesional tissue. In this case, the morphologic distinction between ADH and low-grade DCIS may be ambiguous.

Other investigators $(4,5)$ have reported that in the setting of atypia, NCBB are associated with a significant rate of false negatives. In view of these findings, as well as the considerations above, some have recommended open excisions in all patients with atypical lesions on NCBB. However, to date there are a limited number of published studies that critically evaluate the significance of atypia in these samples.

It is the aim of this study to describe factors that predict underdiagnosis (i.e., of DCIS and invasive carcinoma) among a consecutive set of patients with atypical lesions on NCBB who subsequently went to open excision. Accordingly, we subclassified atypical lesions histologically and estimated their extent to determine whether there are pathologic factors that predict for association with unsampled or partially sampled malignancy. We also evaluated pre- and postbiopsy mammograms to determine whether radiographic findings could potentially be useful in predicting the likelihood of missing DCIS on NCBB.

\section{MATERIALS AND METHODS}

Surgical pathology reports of NCBB performed at Harper University Hospital and Karmanos Cancer Institute between January 1997 and June of 2001 with a diagnosis of atypia $(n=258)$ were retrieved. Only those NCBB having a subsequent open excision at our institution were further evaluated ( $n=$ 185). Patients with a previous or concurrent ipsilateral diagnosis of either DCIS or invasive carcinoma were also excluded, resulting in a group of 167 cases. On the basis of the review of the original pathology reports and slides, each NCBB specimen was assigned to one of the following four groups: Group I, ALH ( $n=24)$; Group II, ADH with minimal cytologic atypism ( $n=90)$; Group III, atypia, other (9 columnar, 2 apocrine, 11 papillary); and Group $\mathrm{IV}, \mathrm{ADH}$ with severe/borderline cytologic features $(n=31)$.

NCBB were performed for suspicious microcalcifications $(n=141)$ or masses $(n=26)$ detected on screening mammograms. The stereotactic NCBB were performed on a Fisher stereotactic table using an $11 \mathrm{G}$ Mammotome probe. The calcifications were localized, and 8-41 (average, 20) core biopsies were taken, followed by placement of "clips" to designate the biopsy site. All stereotactic NCBB were placed on a Petri dish divided into quadrants corresponding to the localization of the microcalcifications, which were confirmed on specimen ra- diographs. The tissue from each quadrant was placed in separate cassettes, permitting directed recutting of tissue blocks if the initial sections did not reveal the microcalcifications sampled. Ultrasound-guided NCBB $(n=26)$ were performed using a $14 \mathrm{G}$ Bard disposable core biopsy needle, obtaining 2-10 (average, 5) biopsy cores. After processing with zinc-formalin and paraffin embedding, five $H \& E$-stained levels were routinely prepared on all tissue blocks.

The criteria we employed for diagnosis of atypical hyperplasia are those of Page et al. (2) and of Page and Rogers (6). These lesions consisted of epithelial proliferations that exhibited some, but not all, of the diagnostic criteria for DCIS (or LCIS), including both cytologic and architectural features. In this study, particular emphasis was placed on cytologic features of atypical ductal hyperplasias. See Figure 1. The majority of ADH cases ( $n=90$, Group II) showed a complex, cribriform-like architecture comprised of cells with small, uniform normochromatic nuclei. A subset of ADH cases ( $n=31$, Group IV) exhibited more pronounced nuclear alterations, with enlargement and hyperchromasia of degree less than malignant. Although none had comedo necrosis or unequivocally malignant nuclei, the diagnosis of $\mathrm{ADH}$ (as opposed to DCIS) reflected, in large part, the microfocal nature of worrisome ducts (1-2 foci). Atypical lobular hyperplasia was defined as partially distended lobular units containing monotonous, evenly spaced cells that fill at least one half of the acini (Group I, $n=24$ ).

Our study included a heterogenous set of lesions (Group III, $n=22$ ) that do not enter into the classical descriptive categories of ADH/ALH. Nine of these cases showed so-called columnar alteration with atypia. This entity has been described by several previous investigators, most recently by Fraser et al. (7), and histologically shows dilated acini lined by epithelium having prominent nuclear elongation and luminal cytoplasmic blebs, referred to as "apical snouts." Lesions with atypical columnar alteration were characterized by nuclear stratification and hyperchromasia of degree less than DCIS. In addition, 11 cases in this group exhibited intraductal papillary lesions with complex architectural features in conjunction with cytologic atypism and/or degree of cellularity, which were not unequivocally malignant but were uncharacteristic of straightforward papilloma or papillary hyperplasia. Finally, two biopsies in Group III contained apocrine cells with atypical, but not clearly malignant, cytologic features

Number of atypical foci in NCBB was enumerated retrospectively, as per Ely et al. (4) Pre- and postprocedure mammographic films were compared to evaluate the size of the microcalcification span and the presence of residual microcalcifica- 


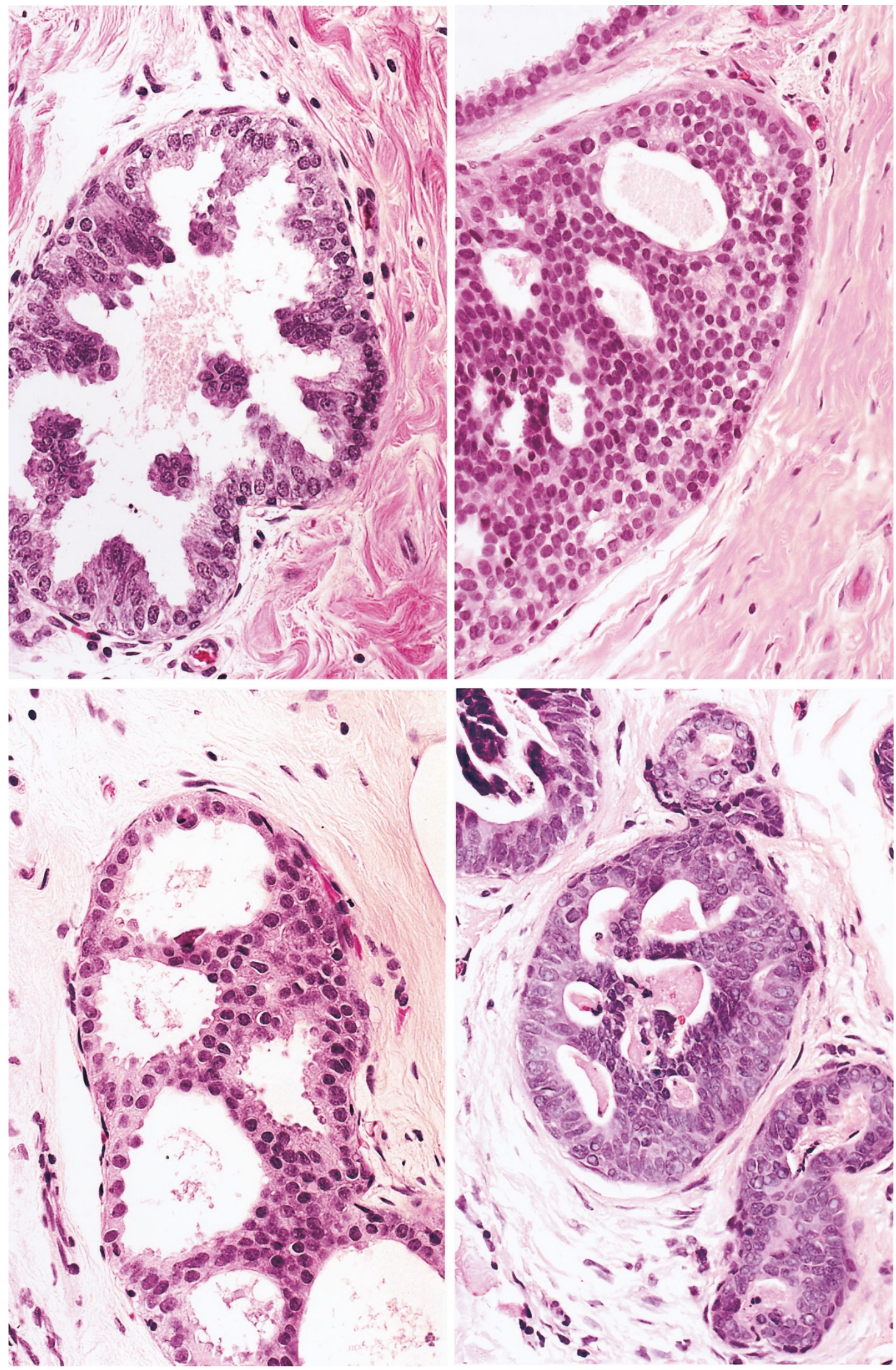

FIGURE 1. Histology of atypical lesions in NCBB. Top left, example of Group II ADH with micropapillary features in which there is circumferential involvement and detachment of cell clusters. Top right, another Group II lesion with cribriform-like architecture; however nuclei are small and partially overlapped. Bottom, Group IV lesions characterized by considerable nuclear atypism and complex architecture. A definitive diagnosis of DCIS is precluded by the focal nature of these changes in our NCBB series. 
tions following biopsy. The amount of residual microcalcifications were represented as a percentage of microcalcifications removed during the biopsy procedure, as evaluated by a radiologist (MR) without knowledge of histologic diagnoses.

Slides from post-NCBB excisions were reviewed retrospectively (by $\mathrm{MB}$ and $\mathrm{DV}$ ) to confirm original diagnoses and to assess the relationship between the location of the core biopsy cavity and residual atypical or malignant lesions (i.e., within $1 \mathrm{~cm}$ of organizing fibrosis versus outside of biopsy perimeter). Per routine, the tissue blocks from excisions performed for residual calcifications were radiographed before sectioning. Those containing calcifications were routinely sectioned at four levels.

\section{RESULTS}

Overall, 36/167 (21.5\%) of patients had DCIS ( $n=$ 32) or invasive carcinomas $(n=4)$ in subsequent open excisions. Patients with DCIS or invasive neoplasms in open excisions were significantly older (60.9 versus $54.3 \mathrm{y}, P<.01$ ). The distribution of open biopsy findings by histologic group is summarized in Table 1.

Twelve percent of open excisions from Groups I-III ( $8 \%$ of Group I, $9 \%$ of Group II, and $27 \%$ of Group III) contained lesions more severe than atypia; 15 of these were DCIS, and 1 was an invasive (tubular) carcinoma, $0.3 \mathrm{~cm}$ in diameter. DCIS was low grade in 13 of $15(87 \%)$ cases, and $60 \%(n=9)$ were $\leq 5 \mathrm{~mm}$. In four, the DCIS size ranged between 1 and $1.5 \mathrm{~cm}$, and in two, it diffusely involved the excision specimen (i.e., $>2.0 \mathrm{~cm}$ ). The extent of atypia in the Group I-Group III NCBB samples was relatively limited (Table 2); $82 \%$ had less than two

TABLE 1. Diagnoses of Open Excisional Biopsies vs. NCBB Group

\begin{tabular}{cccc}
\hline \multirow{2}{*}{$\begin{array}{c}\text { NCBB } \\
\text { Group }\end{array}$} & DCIS or Invasive & ADH & Benign \\
\cline { 2 - 4 } & $2(8 \%)$ & $20(15 \%)$ & $2(1 \%)$ \\
I $(n=24)$ & $8(9 \%)$ & $44(32 \%)$ & $38(28 \%)$ \\
II $(n=90)$ & $6(27 \%)$ & $6(4 \%)$ & $10(7 \%)$ \\
III $(n=22)$ & $4(36 \%)$ & $3(27 \%)$ & $4(36 \%)$ \\
Papillary & $2(22 \%)$ & $3(33 \%)$ & $4(44 \%)$ \\
Columnar & 0 & 0 & $2(100 \%)$ \\
Apocrine & $20(63 \%)$ & $8(26 \%)$ & $3(10 \%)$ \\
IV $(n=31)$ & & & \\
\hline
\end{tabular}

separate foci, and only $4 \%$ had more than four foci. The number of atypical foci in NCBB was weakly correlated with the presence of DCIS in subsequent open biopsy (DCIS group, 5/14 had $>2$ foci in NCBB; no-DCIS group, 4/35 had $>2$ foci in NCBB, $P=.05$ ).

Examination of the open excisions from Groups I-III revealed that the NCBB cavity was immediately adjacent to the more severe lesion in 14/16 (88\%) cases, in keeping with sampling error/artifact. In one case we were not able to define the relationship between the biopsy site and the areas of DCIS. The tubular carcinoma was clearly not located adjacent to a biopsy site and was thus considered an incidental finding in the excisional biopsy, in keeping with a field effect alteration. A background of focal $\mathrm{ADH}$ was found in $75 \%(12 / 16)$ of the excisions with DCIS, compared with $51 \%$ of the open biopsies that lacked more severe lesions $(P=\mathrm{NS})$.

Group IV, the category with worrisome/borderline cytologic features, was significantly different than Groups I and II. Subsequent open excisions showed 20/31 (63\%) with more severe lesions (see Table 1). Twelve of these $(60 \%)$ were low-grade DCIS, 2 (10\%) were intermediate-grade DCIS, 3 (15\%) were high-grade DCIS, and 3 (15\%) were invasive carcinomas. In cases with DCIS only, the size measured $\leq 5 \mathrm{~mm}$ in 10 (58\%), between 0.5 and 1.5 $\mathrm{cm}$ in 5 (29\%), and $\geq 2 \mathrm{~cm}$ in 2 (12\%). The invasive lesions measured $0.3,0.6$, and $1.3 \mathrm{~cm}$, respectively.

Eighteen of the 20 excisions $(90 \%)$ containing DCIS or invasive neoplasm from Group IV had histologically confirmed biopsy cavities. In all but one of the cases, the biopsy site was immediately adjacent to the more severe lesions. In one, the DCIS was located immediately adjacent to the biopsy cavity, but the invasive neoplasm was located $\geq 2$ $\mathrm{cm}$ away and was thus deemed an incidental field effect alteration. In contrast to the cases of Groups I-III, most GIV NCBB $(15 / 24,64 \%)$ had more than two foci of atypia $(P=.001$; Table 2$)$. (Note: In any given case, though, most such foci were not characterized by borderline cytologic features.) Within Group IV, 6/16 NCBB (38\%) having more severe lesions on subsequent open biopsy had four or more foci of atypia, versus $1 / 8$ NCBB (13\%) without DCIS or invasion that had more than four $(P=$ NS).

TABLE 2. Extent of Involvement of NCBB by Atypia vs. Group

\begin{tabular}{|c|c|c|c|c|c|c|}
\hline \multirow[b]{2}{*}{ Number of Foci } & \multicolumn{3}{|c|}{ Groups I-III } & \multicolumn{3}{|c|}{ Group IV } \\
\hline & DCIS & No DCIS & Overall & DCIS & $\begin{array}{c}\text { No } \\
\text { DCIS }\end{array}$ & Overal \\
\hline$\leq 2$ & $18 \%(9)^{*}$ & $63 \%(31)$ & $82 \%(40)$ & $29 \%(7)$ & $8 \%(2)$ & $38 \%(9)$ \\
\hline 3 & $8 \%(4)$ & $6 \%(3)$ & $14 \%(7)$ & $13 \%$ (3) & $21 \%(5)$ & $33 \%(8)$ \\
\hline$\geq 4$ & $2 \%(1)$ & $2 \%(1)$ & $4 \%(2)$ & $25 \%(6)$ & $4 \%(1)$ & $29 \%(7)$ \\
\hline
\end{tabular}

* Numbers in parentheses represent actual number of cases in each category. 
TABLE 3. Histologic Cause of Mammographic Masses in GI-III

\begin{tabular}{lccr}
\hline Histologic Cause & $\begin{array}{c}\text { Number with } \\
\text { DCIS Present }\end{array}$ & $\begin{array}{c}\text { Number without } \\
\text { Presence of DCIS }\end{array}$ & Total \\
\hline Papillary lesion & 5 & 10 & 15 \\
Fibroadenoma & 1 & 4 & 5 \\
Radial scar & 0 & 2 & 2 \\
Fibrocystic changes & 0 & 3 & 3 \\
Duct ectasia & 0 & 1 & 1 \\
\hline
\end{tabular}

Papillomas with atypia comprised 10 of the Group III cases, and there was one additional example of atypical papillary hyperplasia. Most (9/11) NCBB in this group were performed under ultrasound guidance because of presence of a mammographic nodule or mass. Subsequent open excisions in this papillary group showed $36 \%(n=4)$ with DCIS ( 2 cribriform, 1 solid, 1 secretory). Of the atypical columnar lesions $(n=9)$, subsequent excisions showed $22 \%(n=2)$ with DCIS ( 1 high-grade and 1 low-grade micropapillary type) and 33\% ( $n=$ 3 ) with $\mathrm{ADH}$. Both cases with atypical apocrine cells showed benign papilloma.

Mammographically detected masses $(n=26)$ ranged in size from $0.3-2.2 \mathrm{~cm}$ (mean, $0.9 \mathrm{~cm}$; see Table 3). Papillary lesions accounted for the majority of these masses $(15 / 26,58 \%)$. There were five papillomas, apart from Group III lesions previously mentioned, in which atypia was present in surrounding or accompanying ducts. One of these had DCIS in the open excision. Fibroadenomas $(5 / 26$, $19 \%)$, radial scars $(2 / 26,8 \%)$, fibrocystic changes simulating a mass $(3 / 26,11 \%)$, and duct ectasia simulating a mass $(1 / 26,4 \%)$ comprised the remaining cases. (See Table 3). Apart from the papillary lesions already described, open biopsies revealed DCIS in only one of these cases (in which DCIS involved a fibroadenoma).

Mammographic calcification span ranged from $0.2-16 \mathrm{~cm}$ (mean spans per group: Group I, $1.64 \mathrm{~cm}$; Group II, 1.55 cm; Group III, 0.37 cm; Group IV, 2.01 $\mathrm{cm})$. Residual calcifications (i.e., after NCBB) were present in all cases shown to have DCIS on open excision. However, residual mammographic calcification had low specificity because it was present in $71 \%$ of Group I-III cases having negative biopsies. The span of calcifications (on pre-NCBB films) was a significant predicator of DCIS in open biopsy (see Fig. 2). DCIS was present in 6/14 cases with a span of $>2 \mathrm{~cm}$ versus $2 / 59$ cases having a span of $<2 \mathrm{~cm}$ $(P<.001)$.

\section{DISCUSSION}

Much of the literature devoted to ADH/ALH concerns the biological importance of such lesions as markers of constitutional susceptibility for developing breast carcinoma (in either breast; 2, 8). Our

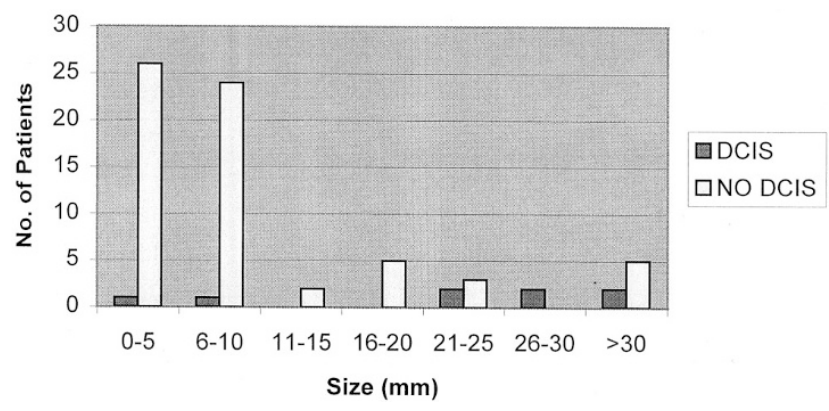

FIGURE 2. Microcalcification span: microcalcification span in Groups I-III is compared in cases with DCIS with cases without DCIS (patients presenting with masses excluded).

study addresses the diagnostic, versus biologic, significance of atypia within the context of a relatively new biopsy technology-the image-guided core biopsy. Because this procedure, in general, samples less tissue than does an "open" biopsy (e.g., guidewire localization), we hypothesized that NCBB with atypia may be associated with a significant false negative rate because of incomplete sampling (particularly in view of the fact that the distinction of atypia from in situ neoplasia is at least partly quantitative in nature). Our results indicate that depending on the particular histologic features of atypia, NCBB is associated with a false-negative frequency of approximately $10-20 \%$. Sampling limitations, as opposed to field effect alteration, account for the vast majority of false negatives (34/36, 94\%).

Previous authors have reported that the frequency of DCIS in excision specimens after a diagnosis of atypical hyperplasia in NCBB ranges from $14-56 \%(4,5,9-11)$. There may be several explanations for this wide variation. First, some earlier studies were performed using $14 \mathrm{G}$ cutting needles $(5,9,11)$, and a limited number of biopsy cores were retrieved per biopsy specimen $(5,9,11)$. As the technology has evolved, many radiologists have increased both the needle bore size, to $11 \mathrm{G}$, and the number of cores that are retrieved during each needle core biopsy procedure $(4,5,10)$. Finally, most centers now obtain routine radiographs of NCBB specimens, after retrieval and before histologic processing, which has been shown to optimize sensitivity by verifying the presence of microcalcifications within the cores and tissue sections $(4,12)$.

At the present time, therefore, patients with atypia in NCBB are generally referred for open biopsy to rule out unsampled DCIS. Because most open biopsies obtained in this setting fail to add diagnostic information, there is a need for criteria that predict the likelihood of unsampled DCIS or invasive carcinoma. Our data suggest at least three findings that imply a greater likelihood for malignancy in open excision. First, more severe ADHparticularly if accompanied by conspicuous cyto- 
logic atypia-was strongly correlated with presence of DCIS in surgical excisions. It is no doubt open to question whether many of our Group IV cases, in view of their atypical nuclear features, could have been initially diagnosed as DCIS on the initial NCBB. We would concede that these cases did, in fact, exhibit "borderline" histologic features. Indeed, in our practice, the pathology reports of these cases typically contain a remark that DCIS cannot be fully excluded or that the findings are "suspicious." Atypical hyperplasia is the appropriate pathologic diagnosis, however, for lesions that are not unequivocally malignant, especially when the number of atypical foci is limited. In view of the finding that most false negatives corresponded to low- grade DCIS, our data underscore the point that NCBB is associated with a significant frequency of undersampling, thus resulting in diagnoses of atypia caused by limited representation.

Retrospective histologic evaluation demonstrated that extent of atypia was quite limited in the core biopsy specimens from our series. This is particularly true of Group II, in which the number of atypical foci exceeded four ducts in $<5 \%$ of cases. However, the number of foci involved by atypical hyperplasia in Group IV was significantly higher than that in Groups I-III (Group IV, average 3.1; $29 \%$ were $>4$ ducts). The threshold of four foci was employed in the recent study by Ely et al. (4), in which they reported that NCBB with four or more atypical foci were significantly more likely to have carcinoma in the open biopsy. If Groups I-IV are combined, our data show that DCIS is present in $7 / 9$ cases with more than four foci, compared with in $11 / 49$ with no more than two foci $(P<.05)$. Clearly, however, the predictive value of this criterion for extent is limited in our series by the small number of cases with more than four atypical foci in Groups I-III. This partly reflects the inclusion of lobular and papillary subsets in our study, which was designed to survey the spectrum of atypical lesions encountered in NCBB.

In patients without significant nuclear atypia, the size of the microcalcification span on the mammogram appears to constitute an alternative measure of disease extent that predicts the potential for DCIS. Our data show that a $<2-\mathrm{cm}$ threshold ruled out $75 \%$ of the DCIS cases in Groups I-III (mammographic masses excluded). It is noteworthy that DCIS size was typically small (most were $<5 \mathrm{~m}$ ) relative to the microcalcification span, further suggesting that sampling error is the major factor that limits sensitivity. These findings are in keeping with prior studies demonstrating that microcalcification span generally exceeds tumor size in DCIS cases. Although it may be useful to combine calcification span with histologic parameters, the limited num- ber of cases in our study precludes meaningful comparisons.

A limited but significant number ( $n=22,13 \%)$ of our cases consisted of atypical lesions that fell outside of the conventional ADH/ALH spectrum and hence were evaluated separately (Group III). Although we do not intend to propose a new system to classify atypical breast lesions, this finding underscores the morphologic diversity of problematic cases that are likely to be encountered in NCBB. This point is noted and explored in a recent review of this topic (13). About half of the Group III cases corresponded to intraductal papillary lesions, most of which were imaged ultrasonographically. In NCBB samples, these atypical papillary lesions were characterized by less conspicuous stroma, increased cellularity, and epithelial atypia. The high proportion of DCIS (36\%) observed in subsequent open biopsies from this subset reflects, in part, the intrinsically low-grade character of papillary DCIS, which may be difficult to distinguish from cellular papillomas, even in open-biopsy samples. The relatively high false-negative rate in this setting may also be attributable to admixtures of DCIS with preexisting benign papillary lesions, which is often observed in such cases. Our data, in conjunction with these considerations, suggest that patients with atypical papillary lesions on NCBB should be strongly considered for open biopsy/excision.

In view of our findings, it seems unlikely that any single factor will be sufficient to determine whether a patient with atypia on NCBB should be spared an open biopsy. Our data imply that several parameters, some pathologic and others clinical (e.g., patient age), predict, albeit imperfectly, a greater likelihood of unsampled DCIS or invasive carcinoma. We have not excluded the utility of other potentially useful clinical indices, including but not limited to patient risk profile (e.g., the Gail Model). Other studies will thus be necessary to definitively resolve the management algorithm for patients with NCBB showing atypia. In the meantime, we would concur with Jacobs et al. (13) that a "low threshold" should be employed in deliberations concerning the decision to perform open excisions on these cases.

\section{REFERENCES}

1. Bonnett M, Wallis T, Rossmann M, Pernick NL, Carolin KA, Segel M, et al. Histologic and radiographic analysis of ductal carcinoma in situ diagnosed using stereotactic incisional core breast biopsy. Mod Pathol 2002;15:95-101.

2. Page DL, Dupont WD, Rogers LW, Rados MS. Atypical hyperplastic lesions of the female breast: a long-term follow-up study. Cancer 1985;55:2698-708.

3. Tavassoli FA, Norris HJ. A comparison of the results of longterm follow-up for atypical intraductal hyperplasia and intraductal hyperplasia of the breast. Cancer 1990;65:518-29.

4. Ely KA, Carter BA, Jensen RA, Simpson JF, Page DL. Core biopsy of the breast with atypical ductal hyperplasia: a prob- 
abalistic approach to reporting. Am J Surg Pathol 2001;25(8): 1017-21.

5. Renshaw AA, Cartagena N, Schenkman H, Derhagopian RP, Gould EW. Atypical ductal hyperplasia in breast core needle biopsies: correlation of size of the lesion, complete removal of the lesion, and the incidence of carcinoma in follow-up biopsies. Am J Clin Pathol 2000;116(1):92-6.

6. Page DL, Rogers LW. Combined histologic and cytologic criteria for the diagnosis of mammary atypical ductal hyperplasia. Hum Pathol 1992;23(10):1095-7.

7. Frazer JL, Raza S, Chorny K, Connolly JL, Schnitt SJ. Columnar alteration with prominent apical snouts and secretions: a spectrum of changes frequently present in breast biopsies performed for microcalcifications. Am J Surg Pathol 1998; 22(12):1521-7.

8. Dupont WD, Page DL. Risk factors for breast cancer in women with proliferative breast disease. N Engl J Med 1985; 312:146-51.
9. Jackman RJ, Nowels KW, Shepard MJ, Finkelstein SI, Marzoni FA. Stereotaxic large-core needle biopsy of 450 nonpalpable breast lesions with surgical correlation in lesions with cancer or atypical hyperplasia. Radiology 1994;193:91-5.

10. Cangiarella J, Waisman J, Symmans WF, Gross J, Cohen JM, $\mathrm{Wu} \mathrm{H}$, et al. Mammotome core biopsy for mammary microcalcification. Cancer 2001;91(1):173-7.

11. Liberman L, Cohen MA, Dershaw DD, Abramson AF, Hann LE, Rosen PP. Atypical ductal hyperplasia diagnosed at stereotaxic core biopsy of breast lesions: an indication for surgical biopsy. AJR Am J Roentgenol 1995;164:1111-3.

12. Liberman L, Evans WP, Dershaw DD, Hann LE, Deutch BM, Abramson AF, et al. Radiography of microcalcifications in stereotaxic mammary core biopsy specimens. Radiology 1994;190:223-5.

13. Jacobs T, Connolly J, Schnitt S. Nonmalignant lesions in breast core needle biopsies. Am J Surg Pathol 2002;26:1095110 .

\section{Book Review}

\section{Kierszenbaum AL: Histology and Cell Biology: An Introduction to Pathology, 640 pp, St. Louis, Mosby, 2002 (\$46.95).}

A few years ago, you may say in the last century, I recommended a histology book written by two British pathologists as the text that I would use to teach histology to medical students. That was then, but today, unquestionably, I would vote for the book presented here. It "reflects much better the spirit of the new trends in medical education in the US," it "appears graphically more appealing and more modern," it "digs in more into the basic cell biology," it "is more conceptual than anatomic." These sound bites under quotation marks are, in essence, what I think about the new book. At the same time, they are also a justification for my switching sides and an apology to my British colleagues for changing allegiances. On the other hand, maybe this will stimulate them, or somebody else, to write in a few years a new and completely different histology that would sweep me off my feeble feet. Faithfulness in emotional matters, said Oscar Wilde, like consistency in intellectual ones is simply a confession of failure and, in 10 years or so, I will need to prove that I am not failing, in more than one manner.

The subtitle of this book is "an introduction to pathology." As such, I would recommend it to all pathology teachers and many a resident interested in learning about the modern views of cell and tissue physiology and dynamic microscopic organ biology. The emphasis is on teach- ing the student how the cells and tissues function, rather than how they appear under the microscope. Having said that, I should add that there is more than enough standard microscopic or electron microscopic morphology to satisfy the old-timers. However, in most instances, the static anatomy has been replaced by functional cell biology, subcellular physiology, and, in many instances, molecular biology. References to pathology are made in dosi refracta, but often enough for my taste. The highlight of the book are the schematic drawings illustrating the main cell components, organization of the tissues, and the functions of various elements. The balance between the new and old stuff has been tipped toward the new, but to reassure the classicist, I have no doubts that the students using this book will know enough microscopic anatomy to understand the sophomore pathology concepts.

This is an exciting new book, and if you are on the curriculum committee of your medical school I recommend that you get a copy and see whether it fits into your teaching program. If it does not, I would suggest that you have a few interdepartmental discussions and reexamine the goals of your teaching system. This might sound a bit cocky on my side, but I strongly feel that this is just what we need for educating the future physicians for the challenges of the 21st century.

\section{Ivan Damjanov \\ University of Kansas School of Medicine Kansas City, Kansas}

